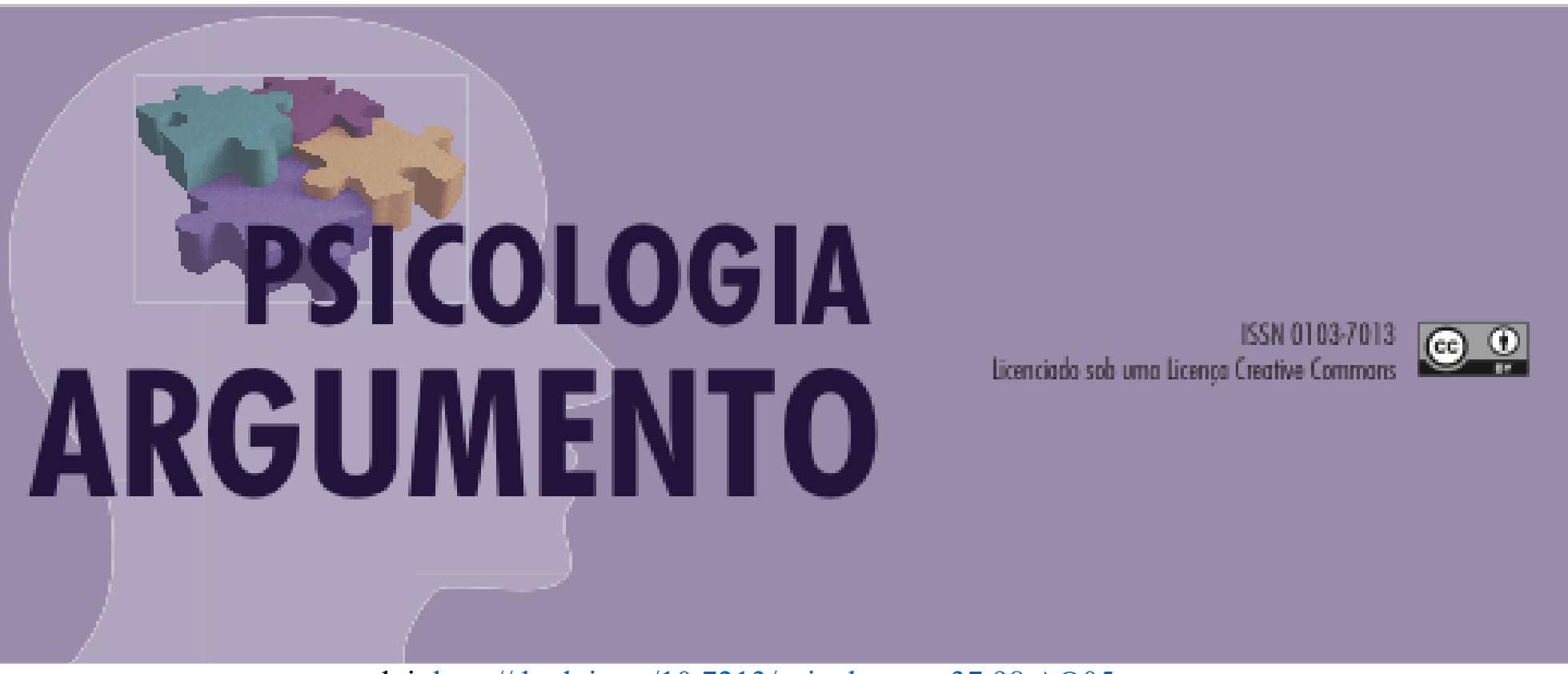

doi: http://dx.doi.org/10.7213/psicolargum.37.98.AO05

\title{
Perfil psicofarmacológico da população masculina atendida na Clínica Escola de uma universidade do Paraná
}

\author{
Psychopharmacological profile of male population attended at the School Clinic \\ of a university of Paraná state.
}

Perfil psicofarmacológico de la población masculina atendida en la Clínica Escolar de una universidad en Paraná

\section{Valéria Teixeira de Souza}

Acadêmica do curso de Psicologia da Pontifícia Universidade Católica do Paraná, PUCPR, Toledo/PR, Brasil.valeriasouza98@gmail.com. Orcid: https://orcid.org/0000-0003-1015-1665

Inara Staub Prochnau

Docente da Pontifícia Universidade Católica do Paraná - PUCPR, Toledo/PR, Brasil. inara.prochnau@pucpr.br. Orcid: https://orcid.org/0000-0002-4128-6944

Tiago Rafael Sausen

Docente do Centro Universitário Univel. Cascavel/PR, Brasil. tiago.sausen@univel.br https://orcid.org/0000-0001-7216-7529

Resumo

Transtornos mentais são alterações temporárias ou duradouras que interferem de forma significativa na vida de muitas pessoas. Uma das formas de tratamento é o uso de psicofármacos, substâncias que podem atuar diretamente em neurotransmissores diminuindo o desconforto/sofrimento do paciente. O objetivo do trabalho foi investigar os psicofármacos utilizados no tratamento de psicopatologias de homens adultos atendidos na Clínica Escola de uma Universidade. Para tanto, foi empregada pesquisa documental (triagens e prontuários) do biênio 2016/2017 e análise comparativa dos dados. Verificou-se que foram atendidos 29 homens com média de idade de 34 anos. As classes de psicofármacos citadas foram antidepressivos, antipsicóticos e ansiolíticos. Onze indivíduos administravam antidepressivos e doze não utilizavam medicação. As subclasses de antidepressivos encontradas foram: inibidores 
seletivos da captação da serotonina, inibidores seletivos da recaptura da serotonina e noradrenalina e antidepressivos atípicos, administrados para o transtorno de ansiedade. Citalopram, fluoxetina e bupropriona eram utilizadas no transtorno de pânico em conjunto com diazepam. Em apenas três casos haviam antidepressivos administrados no transtorno depressivo. Os antipsicóticos (haloperidol e risperidona) estavam sendo administrados para casos de ansiedade. Os resultados corroboram com as informações de que os antidepressivos são medicamentos amplamente utilizados pela população, especialmente no tratamento da ansiedade.

Palavras-chave: Psicofármacos. Antipsicóticos. Antidepressivos. Ansiolíticos.

\begin{abstract}
Mental disorders are temporary or lasting changes that significantly affect many people's lives. Psychotropic drugs, substances that can act directly on neurotransmitters, reducing the patient's discomfort / suffering, can be used as a treatment. The aim of the work was to investigate the psychotropic drugs used in the treatment of adult male psychopathologies treated at in a University School Clinic. A documentary research (screenings and medical records) during 2016/2017 biennium and comparative data analysis were used as method. The population that fitted the criteria was 29 men, having a average age of 34 years. The classes of psychotropic drugs mentioned were antidepressants, antipsychotics and anxiolytics. Eleven individuals administered antidepressants and twelve did not use medication. The subclasses of antidepressants found were: selective serotonin uptake inhibitors, selective serotonin and norepinephrine reuptake inhibitors and atypical antidepressants, administered for anxiety disorder. Citalopram, fluoxetine and buproprione were used in panic disorder combined with diazepam. In only three cases antidepressants were prescribed for depressive disorder. Antipsychotics (haloperidol and risperidone) were prescribed to treat cases of anxiety. The results corroborate with the information that antidepressants are drugs widely used by the population, especially to treat anxiety.
\end{abstract}

Keywords: Psychopharmaceuticals. Antipsychotics. Antidepressants. Anxiolytics.

\title{
Resumen
}

Los trastornos mentales son cambios temporales o duraderos que afectan significativamente la vida de muchas personas. Una forma de tratamiento es el uso de drogas psicotrópicas, sustancias que pueden actuar directamente sobre los neurotransmisores, reduciendo la incomodidad / sufrimiento del paciente. El objetivo del trabajo fue investigar las drogas psicotrópicas utilizadas en el tratamiento de las psicopatologías masculinas adultas tratadas en una clínica de la escuela universitaria. Con este fin, se utilizaron investigaciones documentales (evaluaciones y registros médicos) para el bienio 2016/2017 y análisis de datos comparativos. Se encontró que asistieron 29 hombres con una edad promedio de 34 años. Las clases de drogas psicotrópicas mencionadas fueron antidepresivos, antipsicóticos y ansiolíticos. Once individuos administraron antidepresivos y doce no usaron medicamentos. Las subclases de antidepresivos encontrados fueron: inhibidores selectivos de la absorción de serotonina, inhibidores selectivos de la recaptación de serotonina y noradrenalina y antidepresivos atipicos, administrados para el trastorno de ansiedad. Citalopram, fluoxetina y bupropriona se usaron en el trastorno de pánico junto con diazepam. En solo tres casos se administraron antidepresivos para el trastorno depresivo. Se administraron antipsicóticos (haloperidol y risperidona) para casos de ansiedad. Los resultados corroboran con la información de que los antidepresivos son medicamentos ampliamente utilizados por la población, especialmente en el tratamiento de la ansiedad.

Palabras clave: Psicofarmacéuticos. Antipsicóticos. Antidepresivos. Ansiolíticos. 


\section{Introdução}

Os transtornos mentais são fenômenos que acompanham a humanidade no decorrer de sua evolução sociocultural, de modo que se têm registros e estudos acerca dos mesmos nos diferentes momentos da história, bem como, distintas formas de reações e classificações que foram evoluindo de um período ao outro. Tais fenômenos podem ser considerados como vivências, estados mentais e padrões comportamentais que possuem uma especificidade psicológica, uma vez que as experiências que os doentes mentais experimentam têm uma dimensão própria, não sendo apenas exageros (Dalgalarrondo, 2008).

$\mathrm{Na}$ atualidade é possível observar a significativa presença destes fenômenos psicopatológicos no cotidiano da população em geral, os quais se traduzem nas condições crônicas mais prevalentes, não diferenciando indivíduos com relação à classe social, sexo ou faixa etária (Andrade, Santos \& Bueno, 2004; Kandel, Schwartz \& Jessel, 2003; Kinsley, 2001). Em contrapartida, houve um crescimento científico gradativo nos últimos tempos, alusivos à compreensão da natureza das psicopatologias e também do diagnóstico e práticas curativas (Baes \& Juruena 2017).

Dentre as formas de controle e/ou tratamento para estes transtornos encontra-se a base medicamentosa, mais precisamente, a utilização de psicofármacos, substâncias que atuam nas funções do sistema nervoso central sobre os neurotransmissores reduzindo no paciente o desconforto provocado pela sintomatologia resultante de seu sofrimento psíquico (Dalmolin, Palmeiro \& Flores, 2009). Desta forma, possuem a capacidade de modificar desde o comportamento, humor e percepção, até funções mentais de pacientes que deles fazem uso.

Tendo em vista tais aspectos, esse trabalho buscou, a partir da verificação dos principais transtornos mentais diagnosticados nos pacientes atendidos na Clínica Escola de Psicologia de uma Instituição de Ensino Superior (IES) do estado do Paraná, identificar quais foram às medicações utilizadas para o tratamento dos transtornos, comparando o tratamento proposto com o indicado na literatura. Através dos resultados obtidos, estudantes do curso de Psicologia que realizam estágio no local estudado poderão ter maiores informações a respeito do público por eles atendido, permitindo ampla visão com relação à medicação inserida no tratamento dos pacientes atendidos. 


\section{Objetivo}

Investigar quais foram os tratamentos farmacológicos utilizados no tratamento de psicopatologias em pacientes adultos do sexo masculino atendidos na Clínica Escola de Psicologia durante o biênio 2016/2017.

\section{Método}

A proposta de pesquisa foi enviada ao Comitê de Ética em Pesquisa (CEP) no mês de agosto de 2017, sendo aprovada no mesmo mês e ano sob o número do protocolo PA1715821. Após aprovação, deu-se início à etapa de levantamento de dados, de forma presencial, por meio de pesquisa documental nas fichas de triagens e prontuários da Clínica Escola de Psicologia da IES do estado do Paraná durante o biênio 2016/2017. Foram coletadas as seguintes informações dos pacientes adultos (pacientes com idade superior ou igual a 18 anos), do sexo masculino:

- Sexo do paciente (masculino);

- Idade do paciente (faixa etária);

- Tipo de psicopatologia apresentada pelo paciente;

- Uso de medicação específica para tratamento da psicopatologia diagnosticada (nome do medicamento, dosagem e tempo de uso).

O número de indivíduos analisados que antederam aos critérios estabelecidos para a pesquisa e que tiveram seus dados relativos às psicopatologias e classes de psicofármacos utilizados foi de 29 indivíduos do sexo masculino. O período de coleta ocorreu entre os meses de setembro a dezembro de 2016. Posteriormente, foi realizada a análise dos dados observando as fichas dos pacientes, comparando o tratamento proposto com o indicado pela literatura. Com esses resultados, tabelas foram geradas e analisadas.

\section{Resultados}

Durante o biênio 2016/2017, utilizando o critério de seleção estabelecido, foram atendidos 29 indivíduos, com média de idade de 34 anos. Em algumas fichas e/ou prontuários informações como nome, dosagem e tempo de uso das medicações não estavam devidamente descritos. Nesses casos, a ausência de tais informações ocorreu 
por dois motivos; ou os pacientes realmente não faziam o uso de medicamentos ou não se recordavam do nome e/ou tempo de tratamento.

As fichas e prontuários analisados forneceram dados das classes de medicamentos utilizadas pelos pacientes estudados, cujos resultados encontram-se na Figura 1.

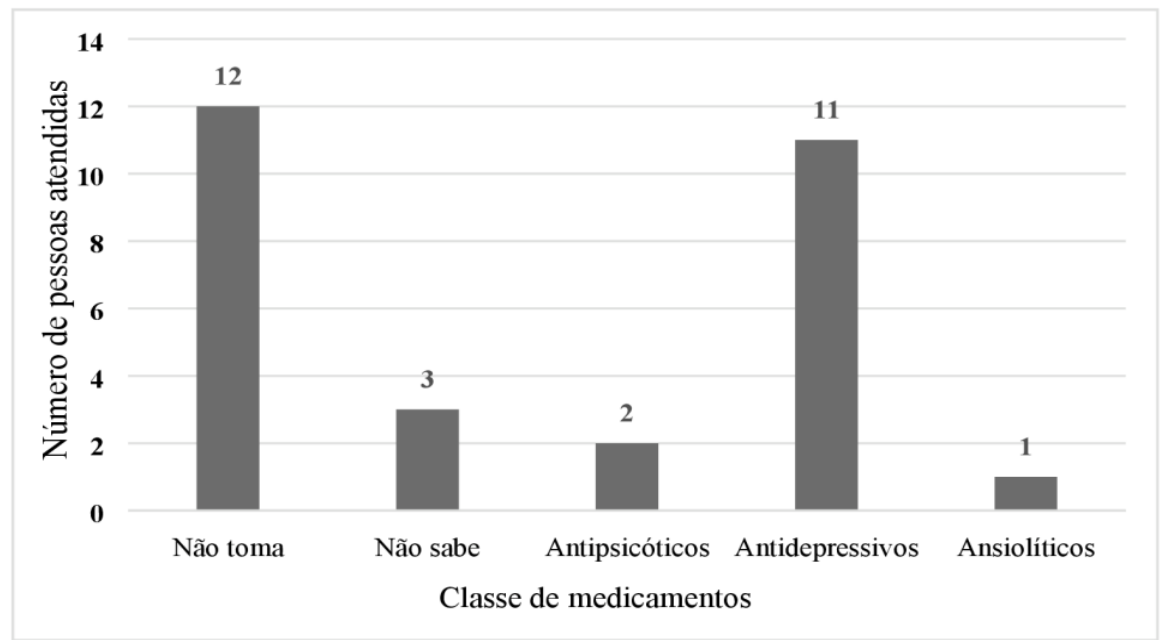

Figura 1. Classe de medicamentos administrados pelos pacientes masculinos atendidos na Clínica Escola no biênio 2016/2017.

Os antidepressivos foram os fármacos mais citados pelos pacientes, seguidos pelos antipsicóticos e ansiolíticos. Por meio dos dados obtidos na análise de fichas e prontuários, foi possível elaborar a Tabela 1, apresentando nome e quantidade dos medicamentos utilizados pelos indivíduos, dentro das classes medicamentosas encontradas. 
Tabela 1. Medicamentos utilizados por pacientes masculinos atendidos na Clínica escola, quantidade de pacientes que fazem uso da medicação e classes medicamentosas.

\begin{tabular}{cc}
\hline Classe Farmacológica & Antidepressivos \\
\hline Medicamento & $\begin{array}{c}\text { Quantidade de } \\
\text { pacientes }\end{array}$ \\
\hline Bromidrato de Citalopram & 1 \\
Cloridrato de Bupropiona & 2 \\
Cloridrato de Fluoxetina & 1 \\
Cloridrato de Sertralina & 2 \\
Cloridrato de Venlafaxina & 2 \\
Oxalato de Escitalopram & 2 \\
Cloridrato de Trazodona & 1 \\
\hline Classe Farmacológica & 1 \\
\hline Haloperidol & 1 \\
Risperidona & Ansiónsicótico \\
\hline Classe Farmacológica & 1 \\
\hline Diazepam &
\end{tabular}

A Tabela 2 apresenta os sintomas/queixas/transtornos descritos pelos pacientes após análise dos dados do ano de 2016. É possível observar que dos 4 pacientes que faziam uso de psicofármacos, 3 faziam monoterapia, sendo os fármacos utilizados oxalato de escitalopram, cloridrato de sertralina e cloridrato de venlafaxina. Apenas um paciente fazia associação de dois medicamentos (cloridrato de bupropiona e cloridrato de trazodona).

Tabela 2. Relação de psicofármacos utilizados por pacientes masculinos atendidos da Clínica Escola e transtorno/queixa/sintoma para os quais foram administrados no ano de 2016.

\begin{tabular}{l|l}
\hline \multicolumn{1}{c|}{ PSICOFÁRMACOS } & \multicolumn{1}{c}{ TRANSTORNO/QUEIXA/SINTOMAS } \\
\hline Cloridrato de Venlafaxina & Transtorno de ansiedade, transtorno depressivo, insegurança. \\
\hline $\begin{array}{l}\text { Cloridrato de Trazodona, } \\
\text { Cloridrato de Bupropiona, }\end{array}$ & Transtorno de ansiedade. \\
\hline Cloridrato de Sertralina & $\begin{array}{l}\text { Autoconhecimento, transtorno depressivo, transtorno de ansiedade, } \\
\text { conflitos familiares devido à orientação sexual. }\end{array}$ \\
\hline Oxalato de Escitalopram & Dificuldade de aprendizagem, transtorno de ansiedade. \\
\hline Não toma & $\begin{array}{l}\text { Suicídio, compreensão de questões naturalizadas, crises de angústia, } \\
\text { autoconhecimento. }\end{array}$ \\
\hline Não sabe & Transtorno de ansiedade, transtorno depressivo. \\
\hline
\end{tabular}


A Tabela 3 apresenta os dados obtidos na análise das fichas de pacientes do ano de 2017. Pode-se observar que apenas um indivíduo fazia monoterapia, sendo oxalato de escitalopram o fármaco citado. Os demais pacientes realizavam a associação de medicamentos. Dentre os indivíduos que não faziam uso de medicação, tanto em 2016 quanto em 2017, as queixas resumiram-se em dificuldades de relacionamento interpessoal, crises de angústia, autoconhecimento, baixa autoestima, entre outros.

Tabela 3. Relação de psicofármacos utilizados por pacientes masculinos atendidos da Clínica Escola e transtorno/queixa/sintoma para os quais foram administrados no ano de 2017.

\begin{tabular}{l|l}
\hline \multicolumn{1}{c|}{ PSICOFÁRMACOS } & \multicolumn{1}{|c}{ TRANSTORNO/QUEIXA/SINTOMAS } \\
\hline & $\begin{array}{l}\text { Relacionamento interpessoal, dificuldades no } \\
\text { relacionamento amoroso, ciúmes, autoestima, transtorno } \\
\text { de ansiedade, dificuldades no relacionamento familiar, } \\
\text { agressão fisica, conflitos com a esposa, conflitos } \\
\text { familiares, término do relacionamento conjugal, crise de } \\
\text { angústia, desemprego, autoconhecimento, crises quanto à } \\
\text { sexualidade, orientação parental e abuso de substância } \\
\text { lítica, autonomia e baixa autoestima. }\end{array}$ \\
\hline Não sabe & Transtorno de ansiedade, transtorno depressivo. \\
\hline Haloperidol e Risperidona & Transtorno de ansiedade e casos de nervosismo. \\
\hline Cloridrato de Venlafaxina & Transtorno depressivo. \\
\hline $\begin{array}{l}\text { Diazepam, Cloridrato de } \\
\text { Fluoxetina, Cloridrato de } \\
\text { Bupropiona e Bromidrato } \\
\text { de Citalopram }\end{array}$ & Transtorno de pânico e fobia. \\
\hline Oxalato de Escitalopram & Desamparo e ideação suicida. \\
\hline
\end{tabular}

\section{Discussão}

Inicialmente é importante salientar que os dados obtidos e que serão analisados nessa pesquisa foram àqueles observados a partir das fichas de triagens e prontuários da Clínica Escola. Tendo em vista que os dados coletados, em sua maioria, foram descritos pelo próprio paciente durante a triagem com os acadêmicos do curso de Psicologia, algumas informações podem não ser exatamente conforme a prescrição médica.

Um fator marcante observado a partir dos resultados refere-se ao uso dos antidepressivos, os quais se destacam significativamente diante das demais classes psicofarmacológicas. Pesquisas apontam que, nos dias atuais, os antidepressivos estão 
em segundo lugar na lista de fármacos mais vendidos para desordens do sistema nervoso, ocupando $6 \%$ do total na categoria (Moraes, 2020). Outro fator a ser considerado é a crescente prescrição e consumo de antidepressivos por parte da população não deprimida para o tratamento de outras morbidades (Vicente, 2015).

Os psicofármacos antidepressivos agem de forma geral sob três problemas relacionados aos neurotransmissores: transmissão baixa de serotonina e noradrenalina; pouca liberação de noradrenalina e dopamina e recaptação rápida de serotonina e noradrenalina (Katzung, 2007; Rang, Dale \& Ritter, 2007; Silva, 2010). Para tentar ajustar tais desordens neurais é necessário o uso dessa classe de psicofármacos, os quais podem ser classificados nas seguintes categorias: antidepressivos tricíclicos, inibidores seletivos da captação de serotonina, inibidores da captação de serotonina e noradrenalina; antidepressivos atípicos e inibidores da monoaminoxidase (IMAO) (Debattista, 2014).

No público pesquisado não foi encontrado dados referente ao uso de antidepressivos das subclasses: inibidores da monoaminoxidase e antidepressivos tricíclicos. As subclasses encontradas foram os inibidores seletivos da recaptura da serotonina (cloridrato de fluoxetina, cloridrato de sertralina, bromidrato de citalopram e oxalato de escitalopram); inibidores da recaptura de serotonina e noradrenalina (cloridrato de venlafaxina) e os antidepressivos atípicos (bupropiona e trazodona).

Segundo as fichas de pacientes, cloridrato de venlafaxina, cloridrato de trazodona, cloridrato de bupropriona, cloridrato de sertralina e oxalato de escitalopram estavam sendo empregados no tratamento de ansiedade; cloridrato de fluoxetina, cloridrato de bupropriona e bromidrato de citalopram foram empregados para tratamento de transtorno de pânico. A respeito dessas informações, Debattista (2014) define que há um índice da prescrição de tais medicações para transtornos de ansiedade, fobia entre outros. No biênio 2016/2017, foram encontrados apenas três casos em que esses medicamentos (cloridrato de venlafaxina e cloridrato de sertralina) estavam sendo utilizados para tratar a depressão.

Conforme os resultados observados, os antidepressivos foram administrados especialmente para casos de ansiedade. Sendo assim, foi possível verificar que este transtorno foi o que mais predominou sobre a população masculina no biênio 2016/2017. Ele é composto por sentimentos de medo e ansiedade excessivos e perturbações comportamentais relacionadas (DSM-V, 2014). O paciente demonstra 
sentir medos vagos e desagradáveis resultantes de um desconforto ou tensão derivada de uma antecipação de perigo, de algo desconhecido ou estranho (Castillo, Recondo, Asbahr \& Manfro, 2000). Um paciente relatou sentir sintomas de angústia, este característico do transtorno de ansiedade.

Pacientes descreveram ser diagnosticados também com transtorno de pânico e fobia, os quais são subclasses do transtorno de ansiedade. A $5^{\text {a }}$ edição do Manual Diagnóstico e Estatístico dos Transtornos Mentais (DSM - V) apresenta dois tipos de fobia: a social e a específica, no entanto, na pesquisa documental não estava especificado qual dos dois subtipos foram apresentados. Quanto a sua etiologia, Braga et al. (2010) apontam que fobia está relacionada a um componente hereditário associado a disfunções em circuitos dopaminérgicos e serotoninérgicos, principalmente para as fobias sociais.

Segundo a afirmativa dos autores acima, a terapia farmacológica comumente prescrita para esses transtornos são os antidepressivos, pois são medicamentos inibidores seletivos da recaptura da serotonina e bloqueadores da recaptação da dopamina e noradrenalina. Tais neurotransmissores (serotonina, dopamina e noradrenalina) são os prejudicados pela disfunção neurobiológica do transtorno. O transtorno de pânico é composto pelos seguintes sintomas:

\footnotetext{
Palpitações, coração acelerado, taquicardia, sudorese, tremores ou abalos, sensações de falta de ar ou sufocamento, sensações de asfixia, dor ou desconforto torácico, náusea ou desconforto abdominal, sensação de tontura, instabilidade, vertigem ou desmaio, calafrios ou ondas de calor, parestesias (anestesia ou sensações de formigamento) desrealização (sensações de irrealidade) ou despersonalização (sensação de estar distanciado de si mesmo), medo de perder o controle ou "enlouquecer" medo de morrer. (DSM-V, 2014, p. 208).
}

Os sintomas, de acordo com Torres, Lima e Cerqueira (2001) possuem uma imprevisibilidade, fato este desagradável e ameaçador ao paciente, pois desencadeia ansiedade antecipatória, comportamento de esquiva de múltiplas situações e dependência de outras pessoas, situações que podem ser limitantes. As disfunções bioquímicas estão relacionadas aos neurotransmissores serotonina e noradrenalina, por isso o uso de antidepressivos (Freitas, 2005).

Quanto aos ansiolíticos, no público pesquisado, foi encontrado apenas o uso do diazepam, um benzodiazepínico que atua seletivamente como agonista nos receptores GABA (ácido gama-aminobutírico), principal neurotransmissor inibitório do sistema nervoso central (SNC), mais especificamente na subunidade $\mathrm{GABA}_{\mathrm{A}}$ (Rang, Dale \& Ritter, 2007). Souza, Canto e Delucia (2014) relatam que os benzodiazepínicos são 
depressores seletivos do SNC, e que as suas propriedades farmacológicas variam desde um efeito ansiolítico até a indução do sono, sem interferir de forma demasiada em outras funções cerebrais. O uso de benzodiazepínicos para o transtorno de pânico deve ser utilizado com cuidado, pois esses fármacos podem causar dependência a longo prazo (Salum; Blaya \& Manfro, 2009).

Os benzodiazepínicos são utilizados no tratamento da ansiedade grave e crônica, bem como ansiedade associada a algumas formas de depressão e esquizofrenia (Golan, Tashjian Jr, Armstrong \& Armstrong, 2014). Levando em consideração que o DSM-V classifica diferentes tipos de ansiedade, não foi possível verificar se os transtornos relatados pelos pacientes eram classificados como ansiedades graves, crônicas ou associadas à depressão ou esquizofrenia, pois essa descrição não estava detalhada nos prontuários.

Segundo levantamento de dados, somente um paciente relatou utilizar antipsicóticos. Segundo a ficha desse paciente, o mesmo fazia uso de antipsicóticos de primeira geração de alta potência (haloperidol) e de segunda geração (risperidona), relatando possuir ansiedade e nervosismo, sem descrição detalhada do por que fazia uso dessas duas medicações.

Os antipsicóticos de primeira geração são inibidores competitivos de vários receptores, mas seus efeitos antipsicóticos refletem o bloqueio competitivo dos receptores D2 da dopamina, provavelmente por isso são medicamentos que causam transtornos de movimento conhecido como sintomas extrapiramidais (SEPs). A segunda geração de antipsicóticos têm menor incidência de SEP, mas são associados com maior risco de efeitos adversos metabólicos, como diabetes. A segunda geração de fármacos deve sua atividade ao bloqueio dos receptores de serotonina e dopamina. Alguns antipsicóticos podem ser usados como adjuvantes aos antidepressivos no tratamento da depressão refratária (Whalen, Finkel \& Panavelil, 2016).

De acordo com Silva (2006), os antipsicóticos são responsáveis por atenuar a intensidade das manifestações psicóticas agudas, sintomas estes, presentes em casos de esquizofrenia, transtorno bipolar em fase maníaca e depressão psicótica. Todavia, levando em consideração que as informações prescritas nas fichas de triagens e prontuários em sua maioria são informadas pelo próprio paciente, sugere-se que este pode não ter informado corretamente o transtorno. 
É importante salientar que a utilização de psicofármacos no tratamento de transtornos mentais, não consegue, em muitos casos, resolver a origem do problema. Xavier et al. (2014) pontuam que o tratamento medicamentoso procura reduzir a sintomatologia prejudicial das patologias psiquiátricas, com o intuito de melhor adaptar o indivíduo à realidade. Em suma, a medicação objetiva melhorar a qualidade de vida tanto do paciente como de seus familiares, sempre avaliando a relação risco-benefício do fármaco, além de avaliar se outros recursos foram devidamente explorados.

Uma característica marcante é que a maioria dos pacientes homens atendidos no biênio 2016/2017 não fazia uso de medicação e/ou não sabia responder, mesmo relatando sofrerem de transtornos, como a ansiedade. Cerioni e Herzberg (2016, p. 598) mencionam que "a procura por atendimento psicológico parece ser, na sua grande maioria, motivada por um sofrimento, um momento de dor, de dúvida e muitas vezes de esperança". Considerando o ponto de vista dos autores, nota-se que há um movimento de desconstrução do preconceito social que predomina em relação ao atendimento psicológico. Os dados trazem indivíduos buscando a psicoterapia como uma primeira alternativa para resolverem questões quanto ao autoconhecimento, desenvolvimento de autonomia e casos de baixa autoestima, resolução de conflitos pessoais e familiares, e até mesmo alguns transtornos psicológicos como ansiedade e depressão.

Schwartz e Sachdeva (2015) apontam que em casos de transtorno de ansiedade e seus subtipos, em alguns casos, apenas a psicoterapia, em especial a Terapia CognitivoComportamental, é considerada tratamento eficaz. No entanto, apenas o tratamento psicoterápico em outros casos não é suficiente. Nesses casos, para que haja sucesso, é necessária a combinação da psicofarmacologia, fato observado em alguns pacientes analisados, pois mesmo fazendo uso de fármacos, tais indivíduos procuraram atendimento psicológico por conta própria, orientação médica ou algum outro fator.

\section{Considerações finais}

A partir dos resultados foi possível verificar que as terapêuticas farmacológicas utilizadas pelos pacientes masculinos de uma instituição particular localizada numa cidade do sul do Brasil referem-se aos antidepressivos, seguidos pelos antipsicóticos e ansiolíticos. O transtorno predominante nesta população foi o transtorno de ansiedade, sendo utilizados para seu tratamento os seguintes antidepressivos: cloridrato de 
venlafaxina, cloridrato de trazodona, cloridrato de bupropiona, cloridrato de sertralina e oxalato de escitalopram.

Os antidepressivos mais utilizados foram cloridrato de venlafaxina, cloridrato de bupropriona, oxalato de escitalopram e cloridrato de sertralina. Dentre os ansiolíticos, somente um fármaco foi citado, sendo o mesmo utilizado para transtorno do pânico e fobia. Os antipsicóticos haloperidol e risperidona, segundo as informações documentais analisadas, estavam sendo administrados para casos de ansiedade.

Todavia, a maior parcela da população masculina atendida não fazia uso de nenhuma medicação ou não sabia responder qual fármaco utilizava. A observação de que os pacientes procuraram atendimento psicológico sem uso de medicação como auxílio na resolução de seus conflitos pode ser considerado um avanço para a prática psicológica, pois por muitos anos a psicologia tem sido considerada uma ciência “elitizada" em virtude de grande parcela da população não ter recursos financeiros para ter acesso aos seus serviços.

É sabido que o trabalho do Psicólogo tem significativa relevância no tratamento de desordens mentais, sendo que em alguns casos não é necessário o uso de medicação. Desta forma, esse novo cenário possibilita o tratamento mais humanizado de acolhimento da dor do indivíduo e redução de "abusos" no uso da medicação, uma vez que os medicamentos representam uma significativa parcela dos gastos públicos com a saúde.

No que concerne a sugestões de novas pesquisas considera-se importante à realização de um estudo similar com o público feminino atendido no local estudado para comparações quanto ao uso de medicação, classes de medicamentos e transtornos psicológicos predominantes. Isso possibilitaria uma visão mais abrangente dos pacientes atendidos.

Não houve limitações durante a execução da pesquisa, mas sim desafios e enriquecimento da aprendizagem. Pesquisar, escrever e aprender sobre Psicofarmacologia dentro da Psicologia é uma atitude um tanto quanto desafiadora, pois a carga horária disponibilizada para esse conteúdo é reduzida quanto comparada às demais matérias. Logicamente, isso varia de universidade para universidade. Desta forma, é, e foi importante para a formação a oportunidade de vivenciar esta experiência. 


\section{Referências}

American Psychiatric Association. (2014). Manual diagnóstico e estatístico de transtornos mentais: DSM - V. [Manual] (5a. ed.). Porto Alegre: Artmed.

Andrade, V.M., Santos, H. \& Bueno, O.F.A. (2004). Neuropsicologia Hoje. São Paulo: Artes Médicas.

Baes, C.W. \& Juruena, M.F. (2017). Psicofarmacoterapia para o clínico geral. Revista Medicina, Ribeirão Preto, 50(1), pp.22-36. Recuperado de http://revista.fmrp.usp.br/2017/vol50-Supl-1/Simp3-Psicofarmacoterapia-para-oclinico-geral.pdf

Braga, J.E.F., Pordeus, L.C, Silva, A.T.M.C., Pimenta, F.C.F., Diniz, M.F.F.M. \& Almeida, R.N. (2010). Ansiedade Patológica: Bases Neurais e Avanços na Abordagem Psicofarmacológica. Revista Brasileira de Ciências da Saúde, Paraíba, 14(2), pp. 93-100. Recuperado de periodicos.ufpb.br/index.php/rbcs/article/download/8207/5320.

Castillo, A.R.G.L., Recondo, R., Asbahr, F.R. \& Manfro, G.G. (2000). Transtornos de ansiedade. Revista Brasileira de Psiquiatria, 22, pp. 20-23. Recuperado de http://www.scielo.br/scielo.php?script=sci_arttext\&pid=S151644462000000600006.

Cerioni, R. A. N. \& Herzberg, E. (2016). Expectativas de Pacientes acerca do Atendimento Psicológico em um Serviço-Escola: da Escuta à Adesão. Psicologia, Ciência e Profissão, 36(3), pp. 597-609. Recuperado de http://www.scielo.br/pdf/pcp/v36n3/1982-3703-pcp-36-3-0597.pdf.

Dalgalarrondo, P. (2008). Definição de psicopatologia e ordenação dos seus fenômenos. Em P. Dalgalarrondo. Psicopatologia e semiologia dos transtornos mentais (p. 2730) (2a .ed.) Porto Alegre: Artmed.

Dalmolin, C.C., Kelling, A., Palmeiro, N.M.S., \& Flores, M.L.P. (2009). A prevalência da psicoterapia e psicofarmacoterapia no Centro Integrado de Saúde e Bem Estar Social de Santa Maria, RS. Revista Eletrônica de Comunicação, Informação \& Inovação em Saúde, Rio de Janeiro, 3(4), pp. 191-197. Recuperado de https://www.arca.fiocruz.br/bitstream/icict/17514/2/11.pdf 
Debattista, C. (2014). Agentes Antidepressivos. Em B. Katzung. Farmacologia Básica Clinica. (pp. 521-526) (12 .ed.). Porto Alegre: Amgh Editora Ltda.

Freitas, C. M. (2OO5). Transtorno do Pânico: Uma análise comportamental. Trabalho de Conclusão de Curso, Graduação em Psicologia, Centro Universitário de Brasília. Brasília.

Golan, D.E., TashJian Jr, A.H., Armstrong, E.J. \& Armstrong A.W. (2014). Princípios de farmacologia do sistema nervoso central. Em D. E. Golan, A. H. TashJian Jr, E.J. Armstrong \& A.W. Armstrong. Princípios de Farmacologia: A base fisiopatológica da farmacologia (pp. 389-389) (3 .ed.). Rio de Janeiro: Guanabara Koogan.

Kandel, E.R., Schwartz, J.H. \& Jessell, T.M. (2003). Principios da Neurociência. Barueri: Manole.

Katzung, B.G. (2007). Farmacologia Básica e Clínica. (10ª ed.) São Paulo: Lange.

Moraes, A. L. (2020) Consumo de antidepressivos cresce 74\% em seis anos no Brasil. Revista Saúde. Recuperado de https://saude.abril.com.br/mente-saudavel/consumode-antidepressivos-cresce-74-em-seis-anos-no-brasil.

Rang, H. P., Dale, M. \& Ritter, J. M. (2007). Farmacologia. (4ª.ed.) Rio de Janeiro: Guanabara Koogan.

Salum, G. A., Blaya, C. \& Manfro, G. G. (2009). Transtorno do pânico. Revista Brasileira de Psiquiatria, Rio Grande do Sul, 31(2), pp.86-94. Recuperado de http://www.scielo.br/pdf/rprs/v31n2/v31n2a02.

Schwartz, T. L. \& Sachdeva, S. (2015). Integrando Psicoterapia e Psicofarmacologia: Desfechos, endofenótipos e os fundamentos teóricos sobre eficácia. Em I.R. Oliveira, T. Schwartz \& S.M. Stahl. Integrando psicoterapia e psicofarmacologia: Manual para clínicos. (pp. 05-05). São Paulo: Artmed.

Silva, P. (2010). Farmacologia. (8a .ed.) Rio de Janeiro: Guanabara.

Silva, R. C. B. (2006). Esquizofrenia: uma revisão. Psicologia USP, 17(4), pp. 263-285. Recuperado de http://www.scielo.br/pdf/pusp/v17n4/v17n4a14.pdf .

Souza, R. L. N.; Canto, A. L. M. \& Delucia, R. (2014) Ansiolíticos e Hipnóticos. In: Delucia, R. Farmacologia integrada: Uso Racional de Medicamentos. [Livro] 5. ed. São Paulo: Clube dos Autores, 2014. Cap. 24. p. 291-293. 
Torres, A. R., Lima, M.C.P. \& Cerqueira, A.T.A.R. (2001). Tratamento do transtorno de pânico com terapia psicodramática de grupo. Revista Brasileira de Psiquiatria, Botucatu, 23(3), pp.141-148. Recuperado de http://www.scielo.br/pdf/rbp/v23n3/a06v23n3.

Vicente, A. (2015). Consumo de antidepressivos entre idosos: evidências do Projeto Bambuí. (Doutorado). Fundação Oswaldo Cruz. Belo Horizonte, MG, Brasil.

Xavier, M. S.; Terra, G. M.; Silva, C. T.; Mostardeiro, S. C. T. S.; Silva, A. A. \& Freitas, F. F. (2014). O significado da utilização de psicofármacos para indivíduos com transtorno mental em acompanhamento ambulatorial. Escola Anna Nery Revista de Enfermagem, Santa Maria, 18(2), pp.323-329. Recuperado de http://www.scielo.br/pdf/ean/v18n2/1414-8145-ean-18-02-0323.pdf.

Whalen, K., Finkel, R., Panavelil, T.A. (2016). Farmacologia ilustrada. (6 ${ }^{\mathrm{a}}$.ed.) Porto Alegre: Artmed. 